\title{
Machines textuelles, machines urbaines
}

Le futurisme et la ville, entre littérature, arts plastiques et architecture

\section{Viviana Birolli}

\section{(2) OpenEdition}

Journals

Édition électronique

URL : http://journals.openedition.org/agedor/3458

DOI : 10.4000 /agedor.3458

ISSN : 2104-3353

Éditeur

Laboratoire LISAA

Référence électronique

Viviana Birolli, « Machines textuelles, machines urbaines », L'Âge d'or [En ligne], 11 | 2018, mis en ligne le 07 janvier 2020, consulté le 17 janvier 2020. URL : http://journals.openedition.org/agedor/3458 ;

DOI : 10.4000/agedor.3458

Ce document a été généré automatiquement le 17 janvier 2020.

L'Âge d'or. Images dans le monde ibérique et ibéricoaméricain 


\title{
Machines textuelles, machines urbaines
}

Le futurisme et la ville, entre littérature, arts plastiques et architecture

\author{
Viviana Birolli
}

« Une automobile rugissante [...] est plus belle que la Victoire de Samothrace»

1 La passion des futuristes pour la machine, en particulier celle de leur fondateur Filippo Tommaso Marinetti, n'est plus à démontrer : du catalogue de l'exposition L'estetica della macchina. Da Balla al Futurismo torinese ${ }^{1}$ à l'ouvrage d'Enrico Crispolti Il mito della macchina e altri temi del futurismo ${ }^{2}$, nous ne comptons plus les écrits critiques et les éditions qui soulignent cet aspect fort connu du premier mouvement d'avant-garde italien.

Il semble plus intéressant, en revanche, d'approfondir les racines, à la fois littéraires et journalistiques, de ce topos dans l'œuvre de Marinetti et de comprendre à quel point ce mythe inspire et influence tous les domaines de la «reconstruction futuriste de l'univers $»^{3}$ : projet de métamorphose moderne et artificielle de l'ensemble du réel qui touche à la fois à l'homme, à ses pratiques, à sa sensibilité et à son lieu de vie, la ville.

3 Les deux manifestes de l'architecture futuriste rédigés, entre 1913 et 1914, par Umberto Boccioni et Antonio Sant'Elia témoignent de l'importance que Marinetti et les futuristes assignaient à la métropole. Celle-ci se fait le théâtre des élans et des mœurs de l'homme nouveau de la modernité, refaçonné par la machine et la vitesse. Pour cette ville destinée aux machines et conçue comme une machine, cette dernière devient ainsi à la fois l'acteur autour duquel le tissu urbain est repensé et la métaphore qui en détermine la logique et la conception.

\section{Le Futurisme : vingt ans d'amour pour la machine}

Voilà tout à coup que deux cyclistes me désapprouvèrent [...] Quel ennui ! Pouah !... Je coupai court, et par dégoût, je me flanquai - vlan! - cul par-dessus tête, dans un fossé... Oh, maternel fossé, à moitié plein d'une eau vaseuse ! Fossé d'usine ! ${ }^{4}$ 
C'est au volant d'une voiture sportive que, sorti de route pour esquiver deux cyclistes à la lenteur passéiste, Filippo Tommaso Marinetti ressort d'un fossé de boue d'usine en homme nouveau de la modernité : loin d'être une simple image poétique, cet épisode est, pour le poète, un rituel baptismal à la haute valeur symbolique, auquel il assigne une place de choix dans son manifeste de 1909.

5 En effet, l'image de la machine fait son apparition très tôt dans ce texte, lorsque, après avoir fermé la porte de la chambre opulente et silencieuse du poète décadent, Marinetti se lance avec ses nouveaux compagnons de bataille dans le joyeux capharnaüm de la ville, dont les grands navires, les locomotives affolées, les énormes tramways et les automobiles affamées constituent aux yeux du poète les emblèmes de modernité les plus puissants qui soient.

6 En somme, à maints égards le culte de la modernité futuriste, tel qu'il est conçu par le fondateur du mouvement, se configure depuis le début comme une «machinolatrie ", où la machine est érigée en symbole de la vitesse et du progrès auxquels les futuristes choisissent de consacrer leur jeunesse, avec un enthousiasme qui n'est pas sans rappeler une quête amoureuse qui aurait troqué l'éternel féminin pour la déesse usine, ou machine ${ }^{5}$.

7 Il s'agit là d'une passion qui traversera le futurisme tout au long de son histoire : des nombreux manifestes qui, dans les années 1910 et 1920, prolongent l'enthousiasme inconditionnel pour la vitesse et la voiture du manifeste de fondation, à ceux des années trente qui mettent à l'honneur les frissons du vol et la nouvelle spiritualité plastique extra-terrestre de l'aérofuturisme ("Manifeste de l'aéropeinture », 1931) 6 . La machine y figure tour à tour comme modèle de l'homme nouveau de la modernité ("Manifeste de l'art mécanique futuriste», 1922) ${ }^{7}$, idole ("Manifeste de l'idole mécanique ", 1925) ${ }^{8}$, être vivant à protéger en tant que vecteur de la rédemption du travail manuel et de l'élaboration d'une nouvelle esthétique («Pour une société de protection des machines. Manifeste futuriste ", 1927) ${ }^{9}$.

Chez le " riche $»^{10}$ Marinetti, cette passion pour les voitures sportives a une origine tout à fait personnelle et précède largement la fondation du futurisme. Le futur fondateur du mouvement chantait déjà les louanges de la voiture en 1905, lorsque dans la poésie « All'automobile » il en célébrait les vertus et le charme dans un style qui n'est pas sans rappeler l'ode amoureuse courtoise :

Dieu véhément d'une race d'acier

Automobile ivre d'espace

qui piétines d'angoisse, le mors aux dents stridentes !

ô formidable monstre japonais, aux yeux de forge

nourri de flamme et d'huiles minérales,

affamé d'horizons et de proies sidérales, je déchaîne ton cœur aux teuf-teuf diaboliques, et tes géantes pneumatiques, pour la danse que tu mènes sur les blanches routes du monde. ${ }^{11}$

\section{Les sources littéraires et journalistiques de la passion « machiniste » du futurisme}

Cette traduction lyrique de l'imaginaire technico-mécanique associé à la voiture s'inscrit d'autre part dans le sillage d'une tradition poétique précise et trouve un 
antécédent direct dans l'œuvre du poète Gabriele D'Annunzio (1863-1938), qui en 1903 glorifiait déjà la machine dans son poème "Maia ». Celui-ci postule une corrélation entre la machine et le corps humain qui, dans les textes successifs de l'auteur, comme le roman Forse che sì forse che no (1910), débouche sur une humanisation croissante des composants de la machine : voici que le moteur s'apparente à un « cœur de métal» au «bruit puissant et constant ", qui « vrombit » et « halète » jusqu'à devenir un "fracas humain ", et la voiture finira par tomber malade après un accident.

Il en est de même chez Marinetti, pour qui la voiture a un cœur qui bat et sort du fossé du manifeste de fondation tel un "grand requin embourbé » qu'une caresse de son maitre suffit pour réanimer et ressusciter. Plus encore, chez ce dernier, cette proximité née sur le terrain de la poésie aboutira à l'utopie d'une véritable fusion organique entre l'homme et la machine, à l'origine d'un nouveau type humain mécanisé, redéfini physiquement et spirituellement par la vitesse de son temps et trouvant sa première incarnation dans la figure mythologique de Gazurmah du roman Mafarka le futuriste. Roman africain (1909), et sa pleine expression dans le manifeste "L'Homme multiplié et le règne de la machine » (1910), où Marinetti proclame :

[...] l'idée de la beauté mécanique, et nous exaltons l'amour pour la Machine [...]. Ce n'est pas là une image, mais presque une réalité, qu'il nous sera facile de contrôler dans quelques années [...]. Il faut préparer aussi la prochaine et inévitable identification de l'homme avec le moteur [...]. Le type inhumain et mécanique construit pour une vitesse omniprésente [...] sera doté d'organes inattendus : des organes adaptés aux exigences d'une ambiance faite de chocs continus. ${ }^{12}$

11 Au-delà des sources poétiques, Marinetti n'hésite pas non plus à puiser dans les sources journalistiques de l'époque pour nourrir son imagerie : en effet, son œuvre et ses prises de position futuristes traduisent souvent, bien que dans un langage et un registre spécifiques, des thèmes qui étaient dans l'air du temps de l'époque, au croisement entre sources littéraires nobles ou anciennes - des pères symbolistes « derniers aimants de la lune » reniés dans «Tuons le clair de lune» (1909) au sources antiques du manifeste de fondation ${ }^{13}$ - et inspirations journalistiques tirées du marché naissant de la littérature et des périodiques grand public.

12 Ainsi, nous savons qu'il était tombé sous le charme de Le Docteur Lerne, sous-Dieu de Maurice Renard (1908) ${ }^{14}$ - où un scientifique créait le premier exemple d'hommemachine en plaçant un cerveau humain dans le moteur d'une voiture - et qu'il suivait de près les premiers vagissements de la science-fiction, dont la première traduction italienne de The time machine ${ }^{15} \mathrm{de} \mathrm{H.} \mathrm{G}$. Wells publiée par Vallardi au début du siècle, où l'on retrouve une description du lien entre temps et espace qui inspirera la réflexion sur les dynamismes d'Umberto Boccioni.

De même, lorsqu'en 1909 il parle d'une « automobile de course avec son coffre orné de gros tuyaux tels des serpents à l'haleine explosive... », il se réfère probablement à la Fiat 130 HP - mieux connue comme F.2 - qui gagna le Grand prix de France en 1907 avec Felice Nazzaro. Ou peut-être à l'une des rares voitures qui franchirent la ligne d'arrivée du périple Pékin-Paris de 1907, rendu mondialement célèbre par l'œuvre de Luigi Barzini, La metà del mondo vista da un'automobile - da Pechino a Parigi in 60 giorni $^{16}$, publié en 1908 en 11 langues. 
Figure 1

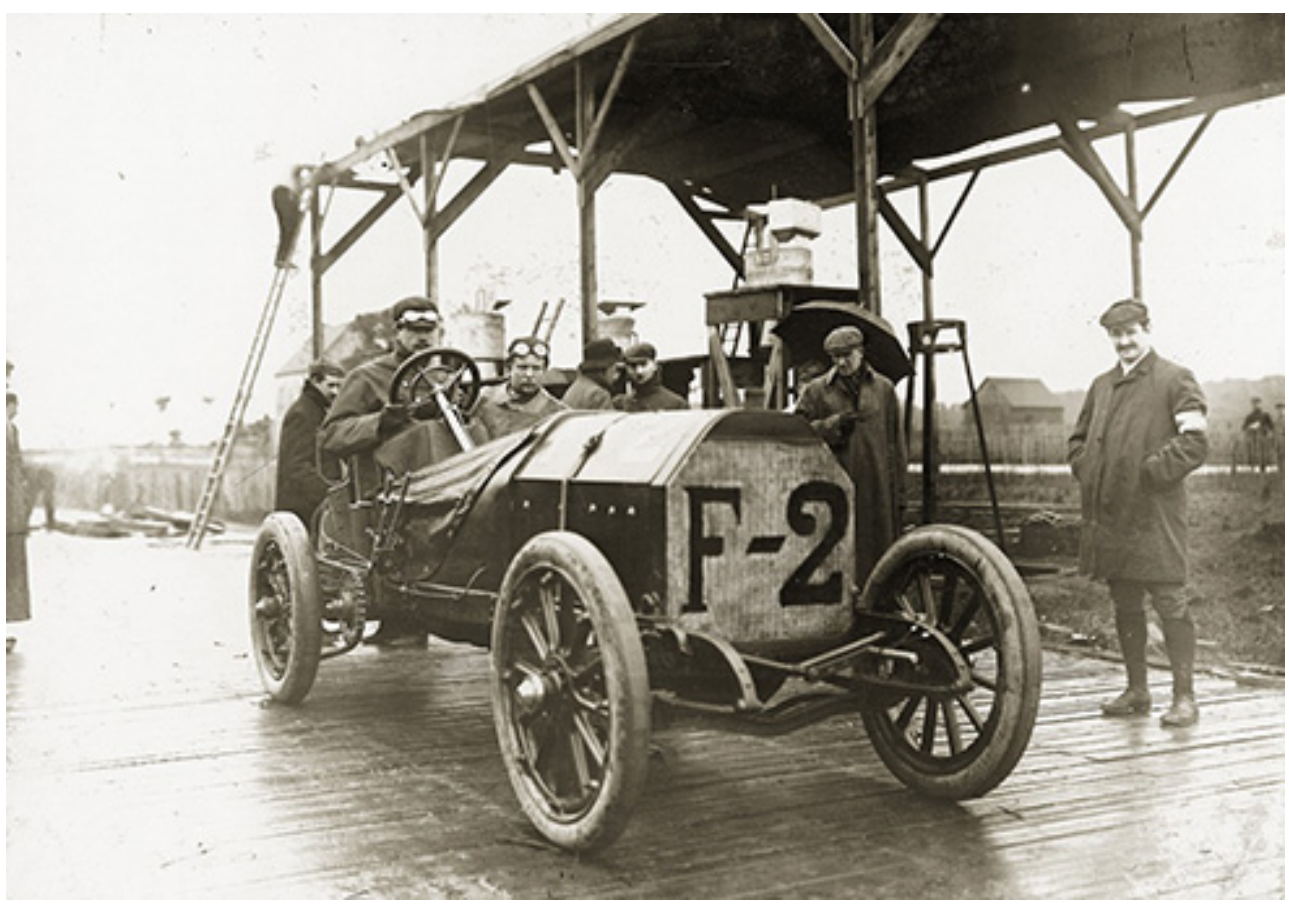

Fiat 130 HP, mieux connu comme F2, gagnant du Grand Prix de France, 1907 belle que la Victoire de Samothrace ", il est en train d'adresser un hommage explicite à Morasso, qui écrivait quatre ans plus tôt : «Quelqu'un affirma jadis que la Victoire ailée et décapitée de Samothrace [...] renferme le vent dans les plis de sa robe [...] lorsqu'il se secoue et piaffe pour le battement excité du moteur, le monstre de fer offre de la même manière une magnifique révélation de force virtuelle $»^{21}$. Au point qu'on pourrait se figurer un jeune futuriste lorsque Morasso parle du jeune homme moderne comme de quelqu'un qui aurait « donné un peu de son cœur au monstre de métal et de feu [mais le monstre lui a donné en retour un peu de sa force et de sa dureté] \» ${ }^{22}$. 
17 Tous les thèmes fondamentaux de l'apologie machiniste des futuristes se retrouvent réunis et anticipés dans cet ouvrage de 1905 : de la défense de la machine contre les critiques passéistes aux modifications anthropologiques entraînées par la vitesse. On y retrouve notamment la première formulation de l'équation fondamentale qui régira la mise en valeur de la machine dans les différents domaines de l'action futuriste : à savoir l'équivalence " machine = vitesse = nécessité = beauté $"$ qui, au fil des différents manifestes, fera, tour à tour, de la machine lancée à toute vitesse, le barème d'une nouvelle nécessité architectonique, le modèle d'une nouvelle sensibilité et le mètre d'une esthétique nouvelle - « Nous déclarons que la splendeur du monde s'est enrichie d'une beauté nouvelle, la beauté de la vitesse. $»^{23}$

18 Clef de voûte de la transition biunivoque entre univers mécanique et humain prônée par les futuristes au nom du dynamisme, la machine s'impose alors comme l'une des principales métaphores structurantes du mouvement italien: moteur de la transformation de l'homme comme de son environnement, modèle pour réimaginer l'homme nouveau et reconstruire la ville et l'univers artificiel de demain autour de ses nécessités nouvelles.

19 Et c'est encore une fois Morasso qui, en 1905, anticipe le lien entre la machine et la ville, au nom d'une révolution dynamique dont la maison moderne est le premier témoin :

Nous n'avons qu'à regarder autour de nous pour trouver les preuves [du fait qu'épargner le temps est devenu la finalité suprême de notre existence]. La maison même que nous habitons en est le premier témoin, avec ses ascenseurs qui nous projettent en une seconde jusqu'au toit, avec ses appareils de chauffage et d'illumination qui permettent d'accomplir instantanément leurs fonctions [...] avec ses téléphones qui suppriment la distance [...], qui ouvrent notre maison immédiatement à toutes les voix du monde. ${ }^{24}$.

20 De cette ville moderne, l'auteur anticipe et détermine à la fois les formes constructives et la logique de fond: de la primauté des grands bâtiments publics et des grands axes de circulation sur les maisons individuelles ${ }^{25}$, à la transformation des places en centres d'irradiation et de gestion de la circulation et du trafic ${ }^{26}$, en passant par l'adieu au piéton $^{27}$, lent héritage du passé destiné à être supplanté par la vitesse des moyens de transport mécaniques.

21 La plupart de ces thèmes rentreront pêle-mêle dans la réflexion des architectes futuristes ; l'œuvre de Morasso ne se borne toutefois pas à démontrer qu'une partie des arguments futuristes, présents dans la littérature et la chronique de l'Italie du Nord de l'époque, étaient dans l'air du temps. Elle anticipe surtout une manière spécifique de sentir la ville comme une machine en mouvement. Lieu d'élection des hommes dynamisés que les futuristes voulaient incarner, ce berceau façonné par la vitesse de la machine et de l'homme au volant deviendra, aux yeux des futuristes, une seconde nature, pour un homme naturellement mécanique et artificiel.

22 De cette ville moderne qui prend comme modèle la « modernissime » Milan et comme contre-modèles les provinciales et passéistes Rome et Venise ${ }^{28}$, Marinetti fournit une première description en 1911, lorsqu'il écrit, dans Le Futurisme :

Dans la ville mécanisée et démocratique [...] les grands édifices stables et riches qui jadis exprimaient l'autorité royale, la théocratie et le mysticisme sont complètement inutiles. Les forces contradictoires des banques [...] les syndiqués [...] la vitesse des communications internationales [...] demandent tout au contraire des 
complexes d'habitation à louer grands et bien espacés, des chemins de fer absolument sûrs, des galeries, des ponts de fer [...]. ${ }^{29}$

\section{Carrà :}

Dans le mouvement futuriste on avait représenté la littérature, la peinture, la musique, la sculpture; il manquait l'architecture [...]. Marinetti était particulièrement inquiet sur ce point [...]. Boccioni remarquait qu'un tel manque dépendait du fait que les architectes cherchaient des éléments concrets et que le futurisme n'avait rien d'attirant à leur offrir. [...] Puis, tout d'un coup, il me vint à l'esprit le nom de Sant'Elia. ${ }^{31}$

Avant que le représentant des futuristes ne songe à approcher le jeune architecte de Côme, son premier choix tombe alors sur un peintre, Umberto Boccioni (Reggio de Calabre, 1882 - Vérone, 1916), à cette époque déjà auteur de deux manifestes consacrés respectivement à la peinture (1910) et à la sculpture futuristes (1912). C'est probablement sur demande expresse de Marinetti que ce dernier rédige, entre la fin de 1913 et le début de 1914, un manifeste de l'architecture qui entretient des liens directs avec celui qu'Antonio Sant'Elia (Côme, 1888 - Monfalcone, 1916) lancera le 11 juillet 1914.

La réception de ces deux textes ne suivra cependant pas l'ordre de leur production : le texte de Boccioni restera un manuscrit inédit jusqu'aux années $1970^{32}$, tandis que celui de Sant'Elia profitera d'une ample fortune éditoriale, due en très grande partie à l'action promotionnelle posthume de Marinetti, qui, dans les années Trente, décida de faire de l'architecte, mort au front alors qu'il n'avait pas encore trente ans, le «champion italien » du rationalisme international.

\section{La ville futuriste, machine pour les machines : deux manifestes de l'architecture futuriste en miroir}

Publiés à moins d'un an l'un de l'autre, et tous les deux mus par le même élan, les manifestes de l'architecture futuriste rédigés par Boccioni et Sant'Elia reflètent néanmoins, dans leurs analogies et leurs divergences, les différents terreaux culturels de leurs auteurs et restituent dans leur ensemble la richesse de la conception futuriste de la ville.

S'inscrivant dans le prolongement des manifestes de la peinture et de la sculpture rédigés peu de temps auparavant par le même auteur, le manifeste d'Umberto Boccioni développe une approche de la ville éminemment visuelle, issue principalement des préoccupations plastiques de l'artiste. Le texte s'ouvre par un état des lieux critique de la situation italienne, typique de tous les manifestes futuristes. L'artiste se lance avec véhémence, et non sans une pointe d'humour, contre l'esclavage de l'architecture vis-àvis des styles anciens et étrangers, définis par des équations ironiques telle «lait de 
vache + chocolat + ennui alpestre $=$ chalet suisse ou art villageois $»^{33}$. Le manifeste se poursuit par la déclaration de la nécessité comme seul essor constructif et principe esthétique de la modernité, ce qui implique un éloignement des instruments modernes de leur origine mimétique :

Un fer chirurgical, un bateau, une voiture, une gare de chemin de fer comportent, dans leur construction, une nécessité de vie qui crée un ensemble de vides et de pleins, de lignes et de plans, d'équilibres et d'équations à travers lesquels jaillit une nouvelle émotion architectonique. ${ }^{34}$

De même, la nouvelle architecture futuriste ne pourra qu'être l'expression d'un nouvel ensemble de besoins et de nécessités liés aux conditions de vie inédites créées par la science et la technologie, qui s'apparentent désormais à de nouvelles forces naturelles à l'ère de l'artificialité :

Il faut que l'architecte jette tout en l'air et qu'il oublie d'être architecte. Qu'il retourne à une nouvelle idée du fondamental [...] l'architectonique que les conditions de vie créées par la science nous imposent comme pure nécessité. ${ }^{35}$

La machine ne peut que jouer un rôle primordial dans ce contexte, car l'équation de nécessité pure "nécessité $=$ rapidité " $^{36}$ (économie + utilité + vitesse), qui s'impose désormais à l'architecte moderne comme règle incontournable, amène naturellement à proscrire toute décoration superflue ou traditionnelle ${ }^{37}$ et à appréhender la maison telle une machine conçue pour fonctionner de manière efficace: "Les pièces d'un édifice doivent donner, comme un moteur, le rendement maximum ${ }^{38}$.

Au-delà de ces indications de méthode générales, l'approche de Boccioni reste toutefois celle d'un artiste prêté à l'architecture : ainsi, la machine est, à ses yeux, avant tout un point de vue privilégié à partir duquel il est possible d'observer la ville autrement, dans une perspective dynamique et accélérée. Ceci offre un nouveau regard porté sur l'espace de la ville qui, d'après Boccioni, doit permettre de tisser un trait d'union entre sensibilités plastique et architectonique, selon l'équation "dynamisme plastique = conscience architectonique dynamique». La sculpture futuriste sera donc une architecture à part entière car, comme l'artiste l'avait déjà avancé dans les notes préparatoires de son manifeste de la sculpture futuriste, l'architectural y est conçu comme une catégorie permettant d'ordonner les choses et les pensées selon leurs lois internes de structure.

De la même manière, pour la plupart des artistes futuristes, la machine est avant tout un outil pour repenser la vision de l'espace urbain, reconfiguré par un dynamisme dont il est nécessaire d'établir les lois structurelles - à savoir les fondements " architectoniques ». Ainsi, si au tout début du mouvement les artistes futuristes se plairont à représenter toute sorte de machines et de mécanismes, dans les années suivantes ils délaisseront souvent cette esthétique machiniste, pour se concentrer davantage sur une étude de l'impact de la machine sur une vision accélérée du monde (Carlo Dalmazzo Carrà, Ce que m'a dit le tram, 1910-1911 ; Luigi Russolo, Dynamisme d'une automobile, 1912-1913). Dès lors, il n'est pas rare que la machine soit représentée deux fois dans les tableaux futuristes, à la fois comme sujet et comme point de vue : vision dissoute de la machine et de la ville depuis une machine en mouvement. La trajectoire de Giacomo Balla résume bien ce parcours de recherche : à partir de 1913, l'artiste peint une centaine de "portraits de voiture » à la recherche des «lois et des lignes-force essentielles" de la vitesse (Vitesse d'automobile, 1913), pour aboutir enfin à des complexes plastiques dynamiques, où la forme sort du cadre du tableau pour se déployer dans l'espace ${ }^{39}$. 
Figure 2

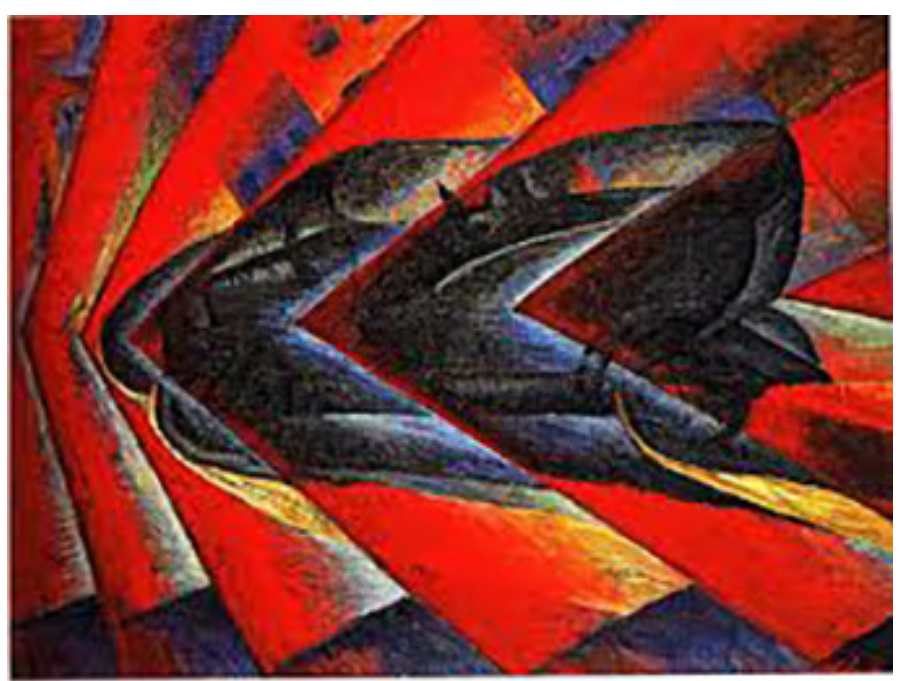

Luigi Russolo, Dynamisme d'une automobile, Composition (Automobile in corsa), 1912-1913 émotion architectonique " - c'est-à-dire d'une nouvelle construction de l'espace façonnée par l'équation "nécessité = rapidité » - éclaircit la racine anthropologique profonde du futurisme en tant que projet de renouvèlement radical de la sensibilité humaine. Comme l'écrit Boccioni en 1910 dans son «Manifeste des peintres futuristes»: «On accusera probablement notre art de cérébralisme tourmenté et décadent. Mais nous répondrons simplement que nous sommes au contraire les primitifs d'une nouvelle sensibilité centuplée [...]. $»^{40}$

\section{qu'être les précepteurs, et la ville moderne le berceau :}

Le futurisme a pour principe le complet renouvellement de la sensibilité humaine sous l'action des grandes découvertes scientifiques. Presque tous ceux qui se servent aujourd'hui du télégraphe, du téléphone, du gramophone, du train, du vélo, de la moto, de la voiture [...] du grand quotidien [...] ne songent pas que tout cela exerce sur leur esprit une influence décisive. ${ }^{41}$, glose Marinetti.

C'est une affirmation commune que de dire, par exemple, que l'invention de la lampe à arc a transfiguré le visage de nos villes. [...] Ce qui ne se souligne jamais assez est à quel point, sous l'impression de tant de changements et des spectacles nouveaux qui en découlent, notre intellect a été lui aussi modifié et, avec lui, toute notre vision de l'univers. ${ }^{42}$, lui fait écho Ardengo Soffici.

De ce point de vue, le rapport institué par les futuristes entre l'homme, la machine et la ville suit deux directions : d'une part, «les nouveaux spectacles de la vie moderne " urbaine imposent de réimaginer le monde et l'homme sous l'égide de la vitesse et de la technologie. D'autre part, c'est l'homme qui se doit de pousser à ses extrêmes conséquences cette métamorphose touchant tant à son environnement qu'à sa sensibilité. En cela, la modernité est pour les futuristes un constat, une croyance, mais aussi une discipline quotidienne.

Boccioni est naturellement l'interprète le plus pointu de cette réflexion perceptive et " architectonique » sur l'espace, construite à la lisière entre arts visuels, arts plastiques et architecture. Parti d'une étude de la déflagration de l'espace entraînée par la force 
« simultanéisante» d'un point de vue en mouvement rapide (« Manifeste technique de la peinture futuriste ", 11 avril 1910 ; La ville se lève, 1910-1911; La rue entre dans la maison, 1911), l'artiste aboutira à une nouvelle vision enveloppante et spiraliforme de l'espace architectonique («Manifeste de l'architecture futuriste», 1913-1914) qui prolongera dans l'espace tridimensionnel les études sur la notion de «dynamisme plastique » qu'il menait depuis 1912 en peinture et en sculpture.

Figure 3

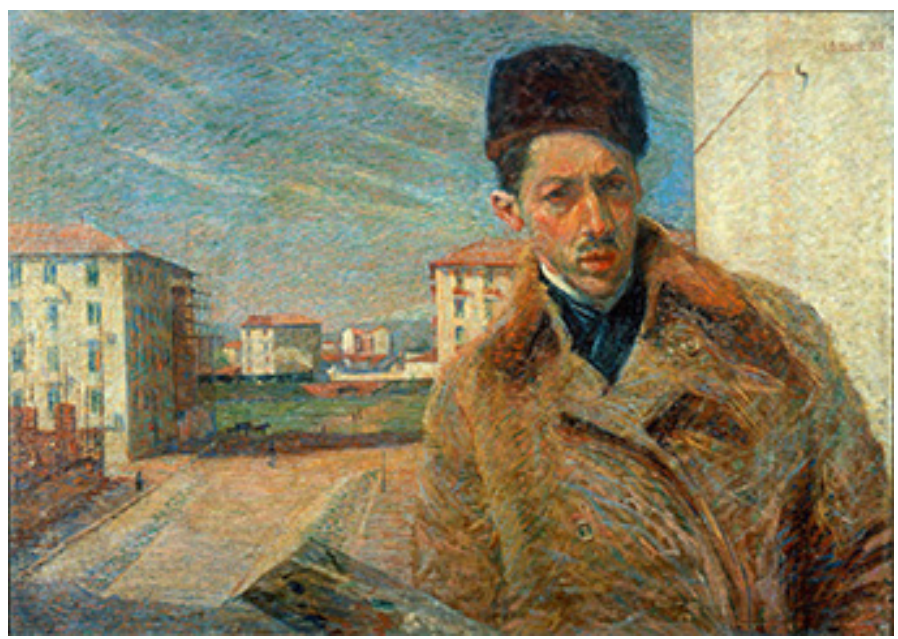

Umberto Boccioni, Auto-portrait, huile sur toile, 1908

Figure 4

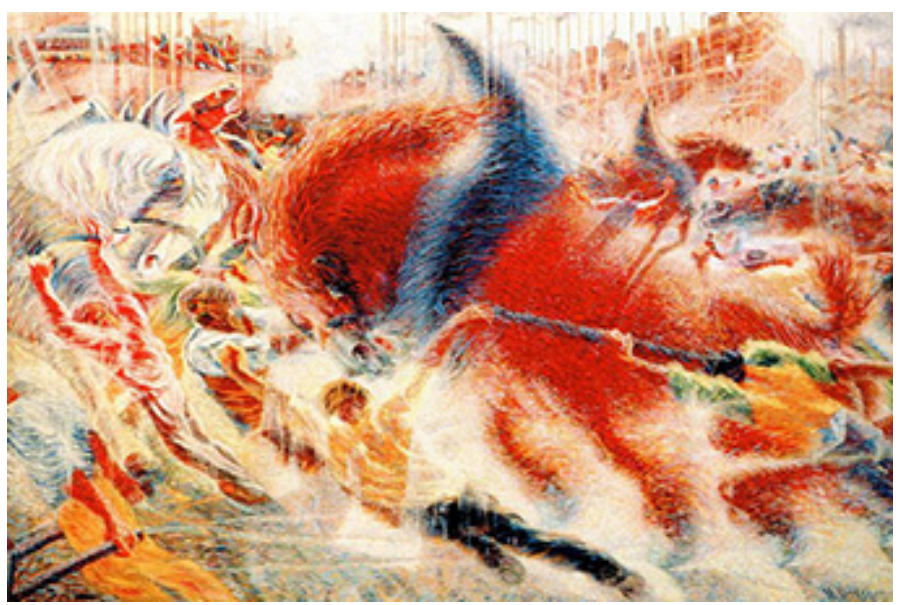

Umberto Boccioni, La ville se lève, huile sur toile, 1910-1911

Nous avons dit qu'en peinture nous placerons le spectateur au centre du tableau [...]. Le cadre de la ville architectonique se transforme lui-aussi en un mouvement enveloppant. Nous vivons dans une spirale de forces architectoniques. ${ }^{43}$

Cet environnement architectonique ne pourra encore une fois qu'être celui de la ville moderne, gigantesque, chaotique, rendue fébrile et éblouissante par les nouveaux prodiges de la technologie :

Jusqu'à hier la construction se déroulait en un sens panoramique et successif. [...]. Aujourd'hui nous commençons à avoir autour de nous un environnement architectonique qui se développe dans tous les sens: depuis les souterrains 
lumineux des grands magasins, depuis les divers étages de tunnels des chemins de

fer métropolitains jusqu'à la montée gigantesque des gratte-ciel américains. ${ }^{4}$

La ville que l'on peut se figurer en lisant ce manifeste pourrait bien être celle de Sant'Elia, tant sont nombreux les points de contact entre ces deux textes: de la conception fonctionnaliste des bâtiments à la valeur esthétique du ciment et du fer, en passant par l'image d'une ville verticale se déployant dans « un ciel infini d'armatures architectoniques $»^{45}$. Caractérisés par un soin technique dû sans doute à la formation classique de l'architecte, les dessins qui accompagnent le manifeste de Sant'Elia constituent néanmoins la toute première, et à maints égards la plus représentative, traduction des imageries de la ville futuriste en véritable projet d'architecture.

Lorsqu'il lance "L'architecture futuriste - Manifeste ${ }^{46}$, le 11 juillet 1914, Antonio Sant'Elia n'a même pas trente ans : approché par Marinetti peu de mois auparavant, il fait confluer dans ce manifeste le fruit d'une série de recherches sur la ville moderne les Dynamismes architecturaux - qu'il menait en privé depuis 1913 et dont il venait de présenter les premiers résultats lors de la « Prima esposizione d'arte del gruppo Nuove Tendenze » à la Famiglia Artistica de Milan (20 mai-10 juin 1914), où il avait choisi d'accompagner ses dessins par un "Message » qui anticipait largement, pour ne pas dire mot à mot, le contenu du manifeste.

À peine un mois après la fin de cette exposition, Sant'Elia sera devenu futuriste et son «Message », un "Manifeste». Pilotés par Marinetti et Decio Cinti, les ajouts et les modifications à la première version du texte du jeune architecte de Côme visent à exacerber les composantes polémiques du "Message ", l'inscrivant dans les codes de l'art de faire les manifestes dont Marinetti se clamait, sans doute à raison, le maître.

41 Le manifeste qui ressort de ces remaniements suit un déroulement on ne peut plus classique pour un manifeste futuriste. Il s'ouvre par un état des lieux polémique de l'architecture de l'époque, où l'auteur se lance contre l'éclectisme ${ }^{47}$ et la fièvre décorative qui faisaient fureur dans l'Italie de ces années grâce à l'œuvre d'architectes tels que Gino Coppedé et Giuseppe Sacconi ${ }^{48}$. À ces fantaisies décoratives mélangeant volontiers exotisme moresque, suggestions moyenâgeuses et élans liberty sur des structures somme toute traditionnelles, Sant'Elia oppose la beauté nue du fer et du ciment, expressions d'un nouveau goût « de la légèreté, du pratique, de l'éphémère et du rapide » qui, chez les hommes modernes, est désormais en passe de supplanter tout « sens du monumental, du lourd, du statique $»^{49}$.

4 À l'aune de cet état de crise qui impose à l'architecture de se séparer de la tradition pour repartir à zéro, la conception d'une maison et d'une ville futuristes se configurent aux yeux de l'architecte comme des problèmes « impérieux » façonnés par l'esprit et les mœurs de l'homme moderne :

La maison et la ville spirituellement et matériellement nôtres, dans laquelle notre

tumulte puisse prendre son essor sans paraître un anachronisme grotesque » seront

la « projection immédiate et fidèle de nous-mêmes. ${ }^{50}$, déclare-t-il.

3 Voici que, une fois de plus, la réalité nouvelle proclamée par les futuristes puise ses racines dans une révolution anthropologique dont l'homme, dans son rapport privilégié avec l'univers artificiel, est le protagoniste. Si l'architecture moderne devra être nouvelle, c'est que la sensibilité moderne impose un tel changement de valeurs constructives et esthétiques. Comme l'explique Sant'Elia :

Le problème de l'architecture futuriste n'est pas un problème de remaniement

linéaire. [...] Il s'agit de forger de toutes pièces la maison futuriste, de la bâtir avec 
toutes les ressources de la science et de la technique [...] une architecture qui ait son unique raison d'être dans les conditions spéciales de la vie moderne, une architecture dont la valeur esthétique réside dans sa correspondance avec notre sensibilité. Cette architecture ne peut être soumise à aucune loi de continuité historique. Elle doit être nouvelle comme notre état d'âme est nouveau. ${ }^{51}$

«nous ressentons » - avant même d'en être conscients - «que nous ne sommes plus les hommes des cathédrales, des palais, des tribunes ; mais ceux des grands hôtels, des gares, des routes immenses, des ports colossaux, des marchés couverts, des galeries lumineuses, des voies rectilignes, des démolitions salutaires $\|^{52}$.

Comme c'était déjà le cas chez Boccioni, le rapport entre cette nouvelle sensibilité et l'environnement qui doit en découler joue sur les deux tables d'un présent à décrypter et d'un futur à bâtir :

[...] comme les anciens prirent leur inspiration artistique dans les éléments naturels, nous - matériellement et spirituellement artificiels - devons trouver l'inspiration artistique dans les éléments du très nouveau monde mécanique créé par nous, dont l'architecture doit être la plus belle expression [...]. ${ }^{53}$

À la différence de l'approche de l'artiste calabrais, chez Sant'Elia ce parti-pris dynamique et mécaniciste se traduit toutefois moins dans un effet de vision qu'en un principe de conception des infrastructures de la ville. Ainsi, dans les dessins de La Città Nuova c'est la ville même qui est appelée à devenir une seule grande machine : elle constitue un dispositif urbain fonctionnant comme une machine en mouvement, conçue dans le but d'optimiser la circulation des machines et des hommes au volant de leurs machines.

Nous devons inventer et refabriquer la ville futuriste à l'image d'un immense chantier tumultueux, agile, mobile, dynamique de toutes parts, et la maison futuriste comme une machine gigantesque..$^{54}$ 


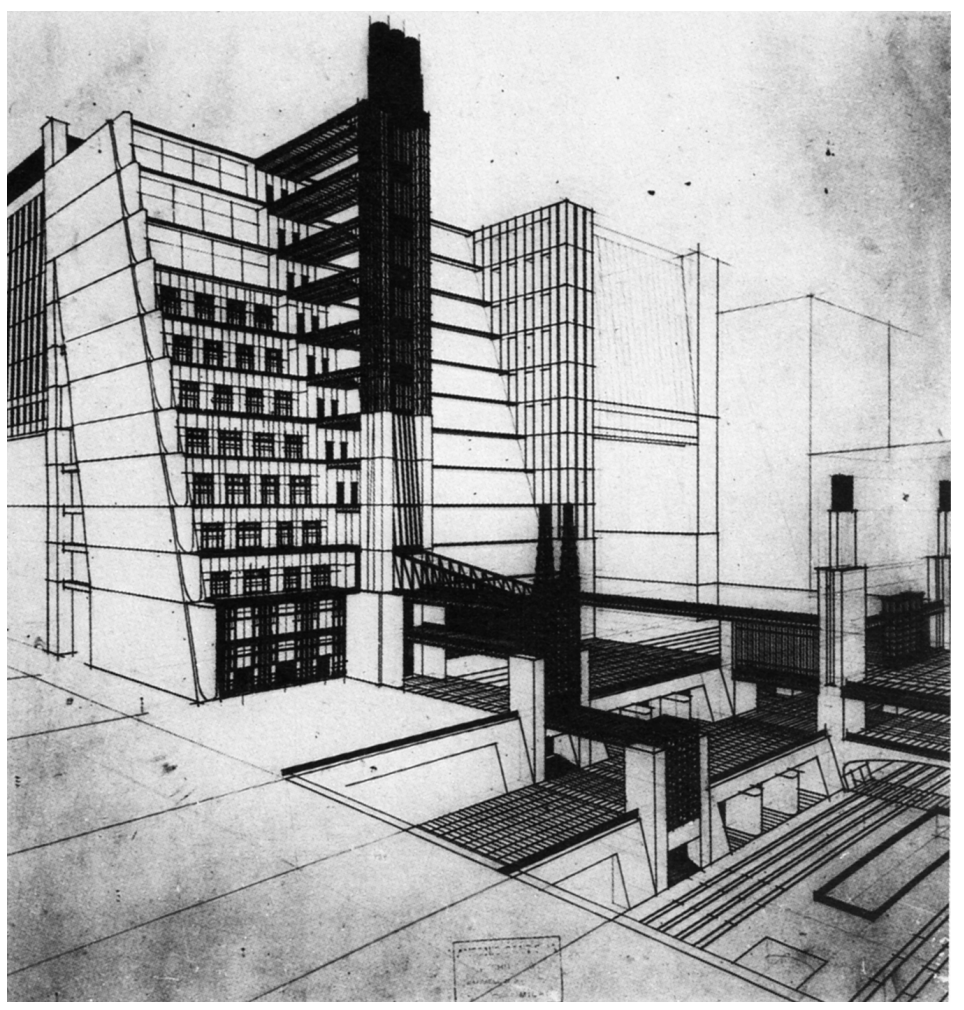

Antonio Sant'Elia, La Città Nuova. Maison en gradins avec ascenseurs, quatre niveaux routiers, 1914, encre et crayon noir sur papier

Emblème d'une logique d'efficacité et de dynamisme touchant à tous les aspects de la vie de l'homme moderne, la machine est dès lors destinée à redessiner le visage de la ville de manière profonde : ainsi, c'est au nom de la supériorité de la machine que les ascenseurs devront remplacer les escaliers vétustes, et le décoratif devra laisser sa place à la maison nue, « riche seulement de la beauté congénitale de ses lignes et de ses formes, extraordinairement laide dans sa simplicité mécanique ». C'est au service de la voiture que la route devra se transformer en un "abîme tumultueux [qui] s'enfuira sous terre par plusieurs étages qui accueilleront le trafic métropolitain et seront reliés [...] par des passerelles métalliques et de très rapides tapis roulants [...] $»^{55}$. C'est en vue d'une circulation optimisée d'unités (hommes, objets ou voitures) que la ville nouvelle n'accordera plus de place aux jardins et aux places des villes «humanistes et artistiques $»^{56}$ des anciens.

Ce n'est donc pas un hasard si la plus grande partie des dessins de La Città Nuova sont des détails d'infrastructures de circulation: les tapis roulants, les routes, les ascenseurs, les viaducs et les ponts sont les véritables protagonistes de la ville nouvelle, pour un homme devenu une unité en mouvement perpétuel, à l'image de la pensée de La Mettrie ${ }^{57}$. Ainsi, chez Sant'Elia la machine devient une métaphore globale, à la fois contenant et contenu de modernité : elle est le cadre théorique d'une nouvelle logique « naturelle et nécessaire» du fonctionnement de la ville, mais elle est aussi un agent actif, qui s'apprête à transformer la ville en une gigantesque infrastructure routière. Une anticipation de la future centralité de la question du trafic que Theo Van Doesburg qualifiera, en 1927, de prophétique. 


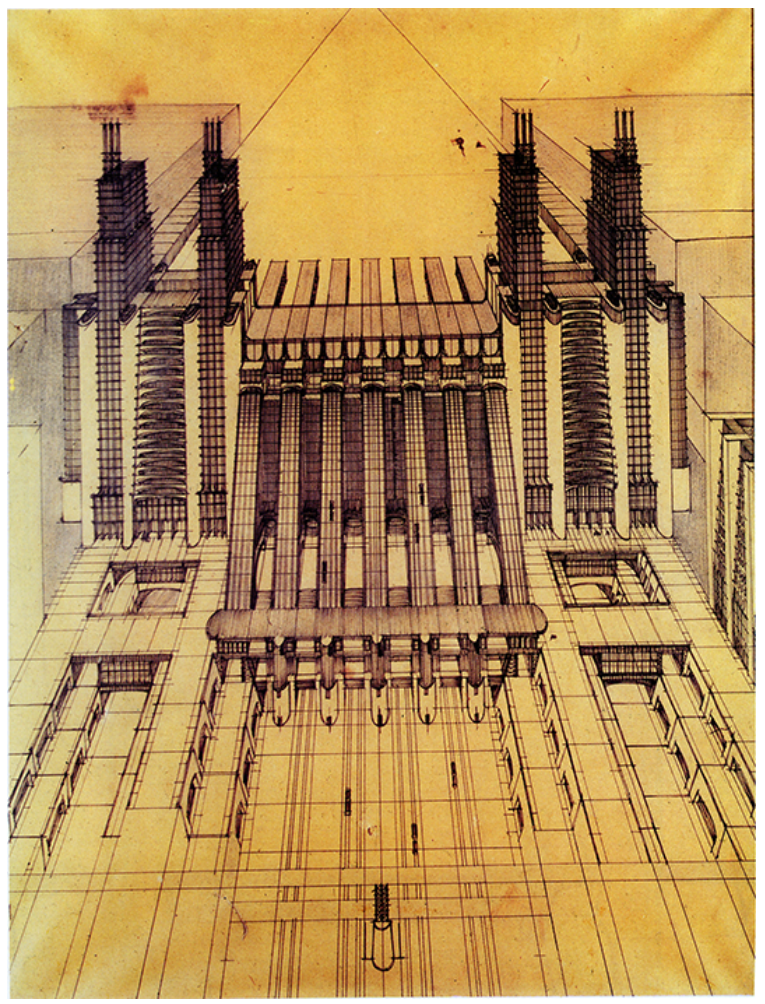

Antonio Sant'Elia, Gare pour trains et avions avec funiculaires et ascenseurs, trois niveaux routiers, 1914, encre noir sur papier

Bien que ce parti-pris véhicule naturellement une approche de la ville à l'échelle du tissu urbain ${ }^{58}$, les dessins de La Città Nuova consistent toutefois presqu'exclusivement en visions panoramiques de bâtiments individuels, voire de portions d'infrastructures de circulation sans aucune hypothèse d'articulation globale. De ce point de vue, ils s'apparentent à des scénographies ${ }^{59}$ : des machines de vision conçues dans le but de définir non seulement le visage de la ville nouvelle de demain, mais aussi le type de regard que l'homme se doit de porter sur elle.

C'est de ce point de vue que se justifient les choix représentatifs propres aux planches de La Città Nuova : du point de vue rabaissé et relativement éloigné aux prises de vue en biais qui dramatisent les angles et dynamisent les lignes, en passant par les coupes supérieures du cadre suggérant l'image d'une verticalité infinie et par une utilisation dramatique de la perspective, dont les raccourcis en contre-point ne sont pas sans rappeler le regard de l'homme minuscule face à l'immensité de la nature du sublime romantique. Autant de choix qui, dans leur ensemble, articulent une rhétorique de la représentation conçue dans le but d'élaborer les lignes de force architectoniques aptes à représenter, à mi-chemin entre projet et scénographie, le visage de la ville nouvelle, mais aussi l'impact visuel qu'elle doit exercer sur ses usagers : ville démesurée, où le hangar, la centrale électrique et la gare se dresseraient, dans leur verticalité, en nouveaux lieux d'un hypothétique culte de la modernité. 
Figure 7

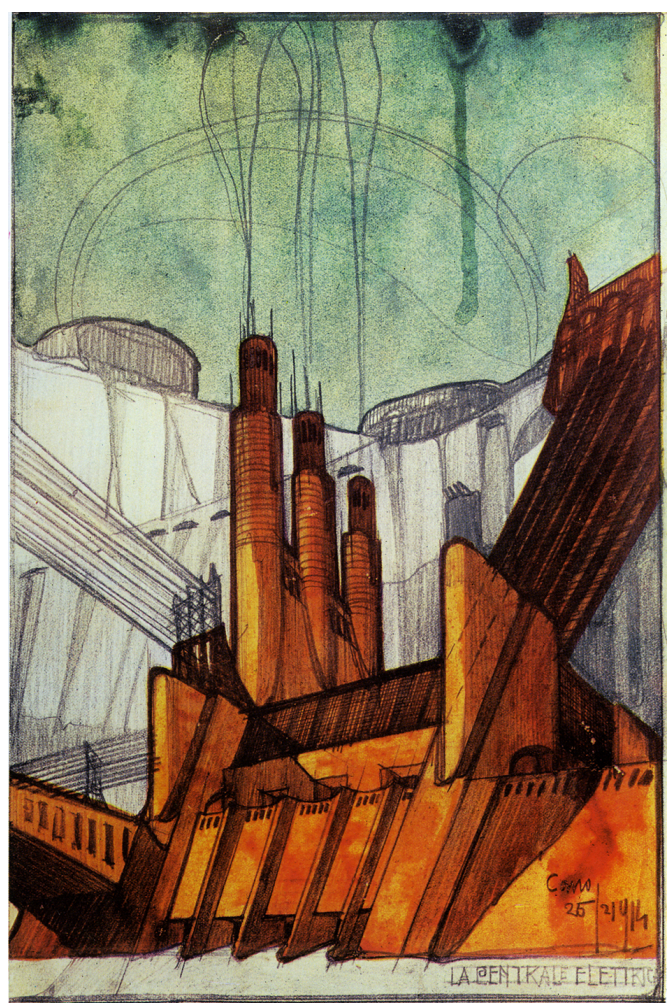

Antonio Sant'Elia, Centrale électrique, 1914, encre noir et gouache sur papier

51 Les circonstances de la genèse de La Città Nuova peuvent aider à mieux comprendre la nature liminaire du projet de Sant'Elia: conçus en vue de la première manifestation publique du groupe éphémère "Nuove Tendenze", originairement ces dessins s'inscrivaient dans le cadre d'une exposition d'art. Un détail qui permet aussi de mieux justifier le décalage entre le caractère visionnaire de La Città Nuova et la nature très classique des rares projets dont l'architecte s'occupa pendant sa courte carrière ${ }^{60}$.

52 L'origine avant tout visuelle et visionnaire de ce projet trouve d'autre part une confirmation dans les études préparatoires - les Dynamismes architecturaux - que l'architecte mène entre 1912 et 1913 : une ample série de prises de vue extérieures de bâtiments immenses et dépourvus d'une destination fonctionnelle claire, qui dans leur nudité austère s'apparentent à autant d'enveloppes vides, pures figures d'idéation spatiale prêtes à accueillir les contenus encore à venir de la modernité. À ce lexique formel ${ }^{61}$ de la modernité, Sant'Elia s'efforcera de fournir une syntaxe avec les études d'infrastructures des planches de La Città Nuova, et un cadre théorique avec son «Manifeste ». 
Figure 8

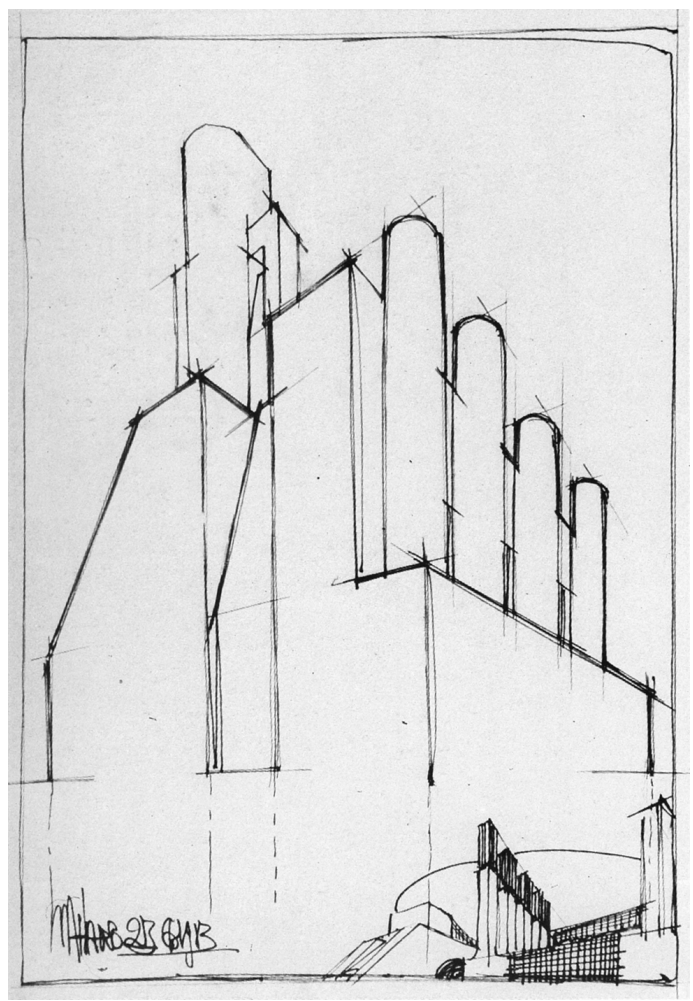

Antonio Sant'Elia, Études pour édifices, 1913, encre noir sur papier

Un «Manifeste » élevé en l'honneur de la machine qui, eu égard aux nombreuses interventions que Marinetti réalise sur le texte, peut lui aussi s'apparenter à une machine : machine textuelle conçue pour accompagner la machine de vision que sont les dessins, fonctionnant comme un mécanisme visant à maximiser l'impact polémique et la charge visionnaire du texte ${ }^{62}$.

\section{NOTES}

1. L'estetica della macchina. Da Balla al Futurismo torinese, catalogue de l'exposition, Turin, Palazzo Cavour, 29 octobre 2004-30 janvier 2005, Milan, Mazzotta, 2004.

2. Enrico Crispolti, Il mito della macchina e altri temi del futurismo, Trapani, Celebes, 1969.

3. Du titre du manifeste lancé par Giacomo Balla et Fortunato Depero en 1915; Giacomo Balla, Fortunato Depero, "La ricostruzione futurista dell'universo", Milan, 11 mars 1915, placard replié ; Giovanni Lista, Futurisme. Manifestes, documents, proclamations, Lausanne, L’Âge d'homme, 1973, p. 202-204.

4. Filippo Tommaso Marinetti, "Le Futurisme", Figaro, 20 février 1909 ; Giovanni Lista, op. cit., 1973, p. 85-89. La version française publiée à la une du Figaro apparaît coupée par rapport au texte en italien et ne mentionne que rapidement l'épisode de l'accident de voiture. Pour une reconstruction de l'ample circulation qu'avait eue le texte intégral du manifeste en Italie durant 
l'année 1909, voir Maria Drudi Gambillo, Teresa Fiori, Archivi del futurismo, vol. I et II, Rome, De Luca, 1958 et 1962 ; Viviana Birolli, Manifesti del futurismo, Milan, Abscondita, 2008.

5. Roberto Tessari, "Le Futurisme et la machine : un mythe d'amour tristanique", Europe, a. 53, n. 551, mars 1975, p. 48-53.

6. Filippo Tommaso Marinetti, Giacomo Balla, Fortunato Depero, Enrico Prampolini, Gerardo Dottori, Benedetta Cappa, Luigi Colombo, "Manifesto dell'Aeropittura", La Gazzetta del Popolo, Turin, 22 septembre 1929 ; publié en français dans Comœdia, 14 février 1931.

7. «Nous sentons mécaniquement. Nous nous sentons construits en acier, nous aussi machines, nous aussi mécanisés ! [...] À partir de la Machine et dans la Machine se déploie aujourd'hui tout le drame humain"; Enrico Prampolini, Ivo Pannaggi, Vinicio Paladini, "Manifesto dell'arte meccanica futurista", Lacerba et Noi, Rome, 20 juin 1922. [nous traduisons].

8. La machine y est présentée comme la véritable idole d'une nouvelle "civilisation mécanique » concrétisée par «tout un complexe matériel et moral qui résoudra enfin l'évolution humaine, avec un perfectionnement définitif »; Fillia, "Manifesto dell'idolo meccanico", L'impero, Rome, juillet 1925.

9. «Les futuristes ont vu les premiers dans la machine non seulement la plus merveilleuse conquête utilitaire de l'humanité, mais aussi la synthèse de la nouvelle esthétique qui changera le visage de la terre au travers d'une reconstruction futuriste entièrement géométrique, mécanique, artificielle et automatique de notre planète "; Fedele Azari, "Per una società di protezione delle macchine. Manifesto futurista", août -septembre 1927, placard replié. [nous traduisons].

10. «Ce jeune riche, doté d'un patrimoine qui pourrait lui assurer les luxes des chevaux, des voitures, des passe-temps coûteux et faciles, des distractions oisives et vaines, a choisi pourtant de sortir de la coutume de la grosse bourgeoisie, de nous rejoindre, nous les artistes et les subversifs et, loin de tout dessein de chantage moral, de consacrer une grande partie de son temps et de ses revenus au LUXE de cette revue [Nda: Poesia]»; Gian Pietro Lucini, "F. T. Marinetti", La Ragione, 27 août 1908. [nous traduisons].

11. Filippo Tommaso Marinetti, “All'automobile”, Poesia, I, n 7, Milan, août 1905, p. 11.

12. Filippo Tommaso Marinetti, "L’Homme multiplié et le règne de la machine", mai 1910, placard replié ; Giovanni Lista, Le Futurisme, Textes et Manifestes, 1909-1944, Ceyzérieu, Champ Vallon, 2015, p. 210-211.

13. Philippe de Lustrac, "Sources antiques des manifestes de Marinetti", Ligeia, $\mathrm{n}^{\circ}$ 69-70-71-72, juillet-décembre 2006, p. 35-36.

14. Maurice Renard, Le Docteur Lerne, sous-Dieu, Paris, Société du Mercure de France, 1908.

15. Herbert George Wells, Un'esplorazione del futuro, Florence, Vallardi, 1902.

16. Luigi Barzini, La metà del mondo vista da un'automobile - da Pechino a Parigi in 60 giorni, Milan, Hoepli, 1908.

17. Dominique Kalifa, Philippe Régnier, Marie-Ève Thérenty et Alain Vaillan (eds.), La civilisation du journal. Histoire culturelle et littéraire de la presse française au XIX $X^{e}$ siècle, Paris, Nouveau Monde, 2011.

18. Cette première publication sera suivie, un an plus tard, par L'artigliere meccanico, qui se rapproche encore plus de la pensée pré-futuriste.

19. Mario Morasso, La nuova arma (La macchina), Turin, Fratelli Bocca, 1905.

20. Des études récentes ont également mis en lumière l'influence de la pensée de Morasso sur D'Annunzio, pour ce qui concerne notamment l'intérêt croissant de ce dernier pour les moteurs ; voir à ce propos Giovanna De Angelis, Stefano Giovanardi, Storia della narrativa italiana del Novecento, vol. I (1900-1922), Milan, Feltrinelli, 2004.

21. Mario Morasso, op. cit., p. 75. [nous traduisons].

22. Ibid., p. 47.

23. Filippo Tommaso Marinetti, op. cit., 1909.

24. Mario Morasso, op. cit., p. 9. [nous traduisons]. 
25. «Plus encore que dans la maison privée, individuelle, ces caractères saillants (précipitation, vitesse, anxiété haletante) s'expriment surtout dans les bâtiments destinés à un usage public, les hôtels, les bureaux, les sièges des journaux. [...] la nouvelle route [...] est un nouvel organe adapté pour de nouvelles fonctions. [...] un canal de transit, c'est un viaduc, c'est un rail sur lequel glisse incessamment le flot infatigable de la vie moderne, c'est une masse en mouvement »; ibid. [nous traduisons].

26. Jadis centre d'affaires et loisirs, "la place moderne - telles les places de l'Opéra ou de la Concorde à Paris, ou la place de la Banque à Londres, ou encore la place du Duomo de Milan - est le centre d'irradiation du mouvement par excellence, le vaste cœur qui permet l'afflux et le reflux de la circulation citoyenne "; ibid., p. 11. [nous traduisons].

27. «Le piéton même [...] commence à être un retardataire et donc source d'embarras ; la roue prime sur les jambes et le char mécanique vante des droits de maîtrise exclusifs »; ibid., p. 12. [nous traduisons].

28. Filippo Tommaso Marinetti, "Contre Venise passéiste", Comœdia, 17 juin 1910, édition italienne datée du 27 avril 1910 ; Giovanni Papini, "Contre Rome et contre Benedetto Croce", discours prononcé lors du meeting futuriste tenu au Théâtre Costanzi de Rome le 21 février 1913, puis publié dans Lacerba, $\mathrm{n}^{\circ} 5,1^{\mathrm{er}}$ mars 1913.

29. Filippo Tommaso Marinetti, Le Futurisme, Paris, Sansot \& Cie, 1911.

30. «Le 11 octobre 1908, après avoir travaillé durant six ans dans ma revue internationale Poesia [...] je sentis, tout d'un coup, que les articles, les poésies et les polémiques ne suffisaient plus. Il fallait absolument changer de méthode, descendre dans la rue, prendre d'assaut les théâtres et introduire le coup de poing dans la lutte artistique »; Filippo Tommaso Marinetti, cité dans Giovanni Lista, op. cit., 1973, p. 16.

31. Carlo Dalmazzo Carrà, La mia vita, Milan, Rizzoli, 1945, p. 99 [nous traduisons].

32. Umberto Boccioni, "L'architecture futuriste - Manifeste", 1913-1914; Zeno Birolli (ed.), Umberto Boccioni. Altri inediti e apparati critici, Milan, Feltrinelli, 1972. La traduction française de ce texte n'a été publiée que très récemment par Giovanni Lista; Giovanni Lista, op.cit., 2015, p. 635-640.

33. Ibid., p. 636.

34. Ibid., p. 637.

35. Ibid., p. 638.

36. Ibid., p. 637.

37. « Nous, nous avons supprimé en peinture et en sculpture tout superflu décoratif, toutes ces préoccupations esthétiques pour le monumental et pour le solennel traditionnel »; Ibid., p. 639.

38. Ibid., p. 639.

39. «Après plus de 20 tableaux sur ce même thème, il comprit que le plan unique de la toile ne permettait pas de donner en profondeur le volume dynamique de la vitesse - se rappelle Depero. Balla sentit alors la nécessité de construire avec des fils de fer, des plans en carton, des étoffes, des papiers velours, etc. le premier complexe plastique dynamique »; Giacomo Balla, Fortunato Depero, "La ricostruzione futurista dell'universo", 1915 ; Giovanni Lista, op. cit., 1973, p. 202-204.

40. Umberto Boccioni, Carlo Dalmazzo Carrà, Luigi Russolo, des peintres de Milan, auxquels se sont ajoutés Giacomo Balla de Rome et Gino Severini, "Manifeste des peintres futuristes ", 1910 ; Giovani Lista, op. cit., 1973, p. 165.

41. Filippo Tommaso Marinetti, "L'imagination sans fils et les mots en liberté", Milan, 11 mai 1913 ; Giovanni Lista, op. cit., 1973, p. 142.

42. Ardengo Soffici, Primi principi di un'estetica futurista, Florence, Vallecchi, 1920 ; Maria Drudi Gambillo, Teresa Fiori, Archivi del futurismo, vol. I, Rome, De Luca, 1958, p. 582. [nous traduisons].

43. Umberto Boccioni, "L'architecture futuriste - Manifeste", 1913-1914 ; Giovanni Lista, op. cit., 2015, p. 639.

44. Ibid., p. 640. 
45. Ibid., p. 640.

46. Antonio Sant'Elia, "L'architecture futuriste - Manifeste”, 1914 ; Giovanni Lista, op. cit., 2015, p. 779-789.

47. «Depuis le XVIII siècle il n'y a plus eu d'architecture. On appelle architecture moderne un mélange grossier d'éléments de style les plus variés, destiné à masquer le squelette de la maison moderne »; Ibid., p. 779.

48. Gino Coppedé (1866-1927) est auteur, entre autres, du quartier Coppedé de Rome (1917), tandis que Giuseppe Sacconi (1854-1905) signe le Monument à Victor-Emmanuel II (1885), mieux connu comme Il Vittoriano de Rome.

49. Antonio Sant'Elia, “L'architecture futuriste - Manifeste”, 1914 ; Giovanni Lista, op. cit., 2015, p. 234.

50. Ibid., p. 780.

51. Ibid., p. 781.

52. Ibid., p. 782.

53. Ibid., p. 783.

54. Ibid., p. 782.

55. Ibid., p. 782.

56. Camillo Sitte, L'art de bâtir les villes. L'urbanisme selon ses fondements artistiques, Paris, Seuil, 1996 [1889].

57. «Le corps humain est une Machine qui monte elle-même ses ressorts; vivante image du mouvement perpétuel »; Julien Offray de La Mettrie, L'homme machine, Paris, Elie Luzac fils, 1748. 58. «Il faut [...] transplanter les problèmes du bon goût en les transférant du domaine du petit profil, du petit chapiteau, du petit portail, à celui plus vaste des GRANDS REGROUPEMENTS DE MASSES, de L'IMMENSE DISPOSITION DES PLANS »; Antonio Sant'Elia, "L'architecture futuriste Manifeste", 1914 ; Giovanni Lista, op. cit., 2015, p. 782.

59. C'est précisément ce caractère visionnaire, pour ne pas dire théâtral, de La Città Nuova qui influencera durablement l'imaginaire cinématographique, à partir de Metropolis de Fritz Lang (1927), et qui trouve des analogies dans les recherches que mèneront, l'un dans le domaine du théâtre, l'autre de l'architecture, Gordon Craig et Hugh Ferriss (The Metropolis of Tomorrow, 1929).

60. Viviana Birolli, “Antonio Sant'Elia et La città Nuova : représenter la ville moderne”, Livraisons d'histoire de l'architecture, $\mathrm{n}^{\circ}$ 32, 2016.

61. Paolo Portoghesi, "Il linguaggio di Sant'Elia”, Controspazio, 1971, p. 30. Dans cet essai, l'auteur analyse de manière détaillée quelques-unes des formes typiques de ce lexique formel: de la pyramide au contrefort, en passant par les lignes obliques et les gradins.

62. À propos du manifeste de Sant'Elia, et plus généralement des manifestes en architecture, voir Viviana Birolli, "Bâtir le manifeste: de La Città Nuova d'Antonio Sant'Elia au manifeste architectural contemporain", Camille Bloomfield et Audrey Ziane (eds.), Le manifeste à travers les arts : devenirs d'un genre indiscipliné, Itinéraires. Littérature, textes, culture, $\mathrm{n}^{\circ}$ 1, 2018 [disponible en ligne, https://journals.openedition.org/itineraires/4223].

\section{RÉSUMÉS}

L'article traite du rapport entre le futurisme et la machine depuis deux axes thématiques : dans une première partie, il s'intéresse aux racines, à la fois littéraires et journalistiques, du topos de la 
machine dans l'œuvre de Marinetti, à l'origine d'un mythe fondateur qui influencera de manière durable tous les aspects et les domaines de la « reconstruction futuriste de l'univers » promue par les futuristes. À la lumière de ce projet de métamorphose moderne et artificielle de l'ensemble du réel, touchant à la fois à l'homme, à ses pratiques, à sa sensibilité et à son lieu de vie, la deuxième partie se consacre à une étude du rôle de la machine dans les manifestes de l'architecture futuriste d'Umberto Boccioni et Antonio Sant'Elia: de la conception d'une ville nouvelle pour des hommes nouveaux à la théorisation d'un nouveau point de vue dynamique pour décrypter le monde, la machine s'y configure comme une métaphore globale, à la fois symbole et moteur, contenant et contenu de modernité.

This article questions the relationship between the Futurism and the machine from two thematic axes: in the first part, it analyses the literary and journalistic roots of the topos of the machine in Marinetti's work, and shows how the machine constituted for the Italian avant-garde movement a founding myth. Influencing all aspects and areas of the "Futuristic Reconstruction of the Universe", the machinist myth will nurture the whole Futurist project of a modern and artificial metamorphosis of reality, touching at the same time man's nature, his practices, his sensitivity and his place of life, the modern city. Thus, the second part of the article develops the role of the machine in Umberto Boccioni's and Antonio Sant'Elia's Futurist Architecture Manifestos: from the project of a new city for new men, to the theorization of a new dynamic point of view to decipher the world, in these Manifestos the machine works as a global metaphor, both container and content of modernity.

\section{INDEX}

Keywords : futurism, manifesto, architecture, machine, modernity, city, Città Nuova, Sant'Elia (Antonio), Boccioni (Umberto), Morasso (Mario)

Mots-clés : futurisme, manifeste, architecture, machine, modernité, ville, Città Nuova, Sant'Elia (Antonio), Boccioni (Umberto), Morasso (Mario)

\section{AUTEUR}

\section{VIVIANA BIROLLI}

Université Paris 1 Panthéon-Sorbonne, HiCSA 